\title{
A FASE FOLICULAR INFLUÊNCIA A PERFORMANCE MUSCULAR DURANTE O PERÍODO DE TREINAMENTO DE FORÇA
}

\section{Charles Ricardo Lopes}

Universidade Metodotista de Piracicaba, Piracicaba, São Paulo, Brasil

\author{
Alex Harley Crisp \\ Universidade Metodotista de Piracicaba, Piracicaba, São Paulo, Brasil
}

\section{Gustavo Ribeiro da Mota}

Universidade Federal do Triângulo Mineiro, Uberaba, Minas Gerais, Brasil

\section{Gabriele Andrilli Avanço}

Faculdade de Americana, Americana, São Paulo, Brasil

\author{
Rozangela Verlengia \\ Universidade Metodotista de Piracicaba, Piracicaba, São Paulo, Brasil
}

\section{Resumo}

O objetivo deste estudo foi avaliar a influência do ciclo menstrual sobre a performance de força muscular. Vinte mulheres treinadas e usuárias de contraceptivos participaram como voluntárias. O treinamento durou 26 dias (12 sessões - segunda, quarta e sexta) e envolveu apenas o exercício supino reto (6 séries de 10 repetições a $80 \%$ de $1 \mathrm{RM}$ e pausas de 60 segundos entre series). Para comparação da performance muscular, foi considerado o volume total de carga levantada (séries x repetições x carga) das sessões referentes: $1^{\circ}$ e $3^{\circ}$ dia (fase folicular), $8^{\circ}$ e $12^{\circ}$ dia (fase ovulatória), $15^{\circ}$ e $26^{\circ}$ dia (fase lútea). Nossos resultados mostraram redução significativa no volume total de carga apenas na fase folicular. Em conclusão, fase folicular influenciou no desempenho da força muscular.

Palavras-chave: Ciclo Menstrual. Força Muscular. Treinamento de Resistência.

\section{Introdução}

O treinamento de força é conhecido como um método efetivo para a melhora da capacidade do sistema neuromuscular, sendo que a manutenção e/ou aumento da força muscular geram resultados benéficos nos mais diferentes aspectos da qualidade de vida (DESCHENES; KRAEMER, 2002; KRAEMER; RATAMESS, 2004).

A variação hormonal observada durante o ciclo menstrual de mulheres em que se observa alternância nas concentrações de estrógeno e progesterona representa um importante aspecto a ser controlado em 
diversos estudos incluindo os que avaliam a performance humana (OOSTHUYSE; BOSCH, 2010). Em relação à razão estrógeno e progesterona o ciclo menstrual é dividido em três fases: i) folicular concentrações baixas de estrógeno e progesterona; ii) ovulatório concentrações elevados de estrógeno e baixos de progesterona e iii) lútea - concentrações elevadas de estrógeno e progesterona durante a fase lútea (CONSTANTINI; DUBNOV; LEBRUN, 2005).

A pílula de contraceptivos orais reduz a variabilidade da duração do ciclo menstrual, proporcionando um ciclo de 28 dias, controlando sistemicamente as concentrações de hormônios endógenos, reduzindo a produção fisiológica de estrógeno e progesterona, inibindo assim a ovulação e prevenindo a gravidez (BURROWS; PETERS, 2007). Em adição, estas são empregadas também no controle dos sintomas prémenstrual, manipulação do ciclo e, o uso tem aumentado entre as mulheres fisicamente ativas (BENNELL; WHITE; CROSSLEY, 1999).

Elevações de estrógeno e progesterona durante o ciclo menstrual podem causar diversos efeitos fisiológicos, incluindo mudanças no sistema termorregulador, respiratório, cardiovascular, renal e que podem por sua vez influenciar o desempenho físico (JENSE DE JONGE, 2003). Alguns estudos têm avaliado a força muscular entre as diferentes fases do ciclo menstrual por meio do teste de 10RM (DIAS; SIMÃO, 2005; LOUREIRO et al., 2011) e 8RM (SIMÃO et al., 2007). Por outro lado, os estudos não abordam a influência do ciclo menstrual durante o período de treinamento.

Assim, o objetivo do presente estudo foi avaliar a influência das diferentes fases do ciclo menstrual (fase folicular, ovulatória e lútea) sobre a performance de força muscular de membros superiores durante a realização de treinamento de força em mulheres jovens treinadas, usuárias de anticoncepcional.

\section{Materiais e Métodos}

Sujeitos

Participaram deste estudo 20 mulheres, (idade: $22 \pm 1$ anos; massa corporal: $56 \pm 5 \mathrm{~kg}$; altura: $162 \pm 1 \mathrm{~cm}$ ) com experiência de $2,2 \pm 0,5$ anos em treinamento de força resistente hipertrófica (de 3 a 5 séries para cada grupo muscular, de 8 a 12 repetições máximas, pausas de 1 a 2 min entre as séries, no mínimo 3 vezes por semana). Os critérios 
de inclusão e exclusão para a participação do estudo foram: (a) ter experiência em treinamento de força (e teste de uma repetição máxima [1RM]) de no mínimo um ano; (b) estar realizando treinamentos com caráter de resistência de força hipertrófica; (c) usuárias de medicamento anticoncepcional no mínimo de um ano; (d) ter um ciclo menstrual regular de vinte oito dias; (f) não estar utilizando suplementos nutricionais.

Todas as participantes responderam questionário de saúde e assinaram Termo de Consentimento Livre e Esclarecido, depois de terem sido informadas sobre o protocolo experimental referente à pesquisa. Previamente a sua realização, este estudo foi aprovado pelo Comitê de Ética Local.

\section{Procedimento Experimental}

Como as voluntárias já praticavam treinamento de força, as mesmas foram orientadas a permanecer uma semana sem treinar a musculatura que seria testada inicialmente neste estudo (membros superiores). Nesta semana, elas apenas realizaram o teste de 1RM para determinação da intensidade do protocolo. Após isso, foram submetidas ao protocolo durante 26 dias, que teve início no primeiro dia da fase folicular, e a partir do qual a força de membro superior foi avaliada nas diferentes fases do ciclo menstrual (folicular, ovulatória e lútea) por meio da alteração da performance da força muscular entre séries.

A queda da performance muscular foi identificada no momento em que a voluntária não completasse o número de repetições estipuladas por série, culminando com a redução da carga inicial proposta. Para análise foi considerado o volume total de carga levantada (séries $\mathrm{x}$ repetições x carga $[\mathrm{kg}]$ ) nas sessões referentes: (i) $1^{\circ}$ e $3^{\circ}$ dia (fase folicular); (ii) $8^{\circ}$ e $12^{\circ}$ dia (fase ovulátória); (iii) $15^{\circ}$ e $26^{\circ}$ dia (fase lútea). As participantes não realizaram outros exercícios de força (membros superiores e inferiores) durante o procedimento experimental.

\section{Determinação da Força Máxima}

A determinação da força muscular máxima foi avaliada no exercício supino reto pelo o teste de 1RM, de acordo com os procedimentos 
descritos por Brow e Weir (2001). O teste foi realizado no $23^{\circ}$ dia fase lútea de cada voluntária, antes do início do estudo.

\section{Protocolo de Treinamento Força}

O protocolo durou 26 dias e envolveu o exercício supino reto com barra livre, que foi realizado com 6 séries de 10 repetições e intervalos de recuperação de 60 segundos entre séries. A intensidade para a realização da primeira série foi de $80 \%$ de $1 \mathrm{RM}$ e, na sequência se necessário, a mesma poderia ser alterada (reduzida) entre as séries de acordo com capacidade de realizar o as 10 repetições e o total de 6 séries propostos. Este protocolo foi realizado três vezes por semana (segunda, quarta e sexta-feira).

Análise Estatística

A normalidade dos dados foi avaliada pelo teste de KolmogorovSmirnov. Para verificar a diferença entre as médias foi utilizado ANOVA One-Way. O teste post hoc empregado foi Turkey. O índice de significância adotado foi pré-fixado em 5\% (Software Graph Pad $\operatorname{Prism}()$.

\section{Resultados}

O volume total de carga levantada (séries $X$ repetições $X$ carga $[\mathrm{kg}])$ foi significativamente menor $(\mathrm{p}<0.05)$ na fase folicular do ciclo menstrual quando comparado com as outras duas fases (Ovulatória e Lútea), as quais não diferiram estatisticamente $(\mathrm{p}>0.05)$ (Figura 1). 


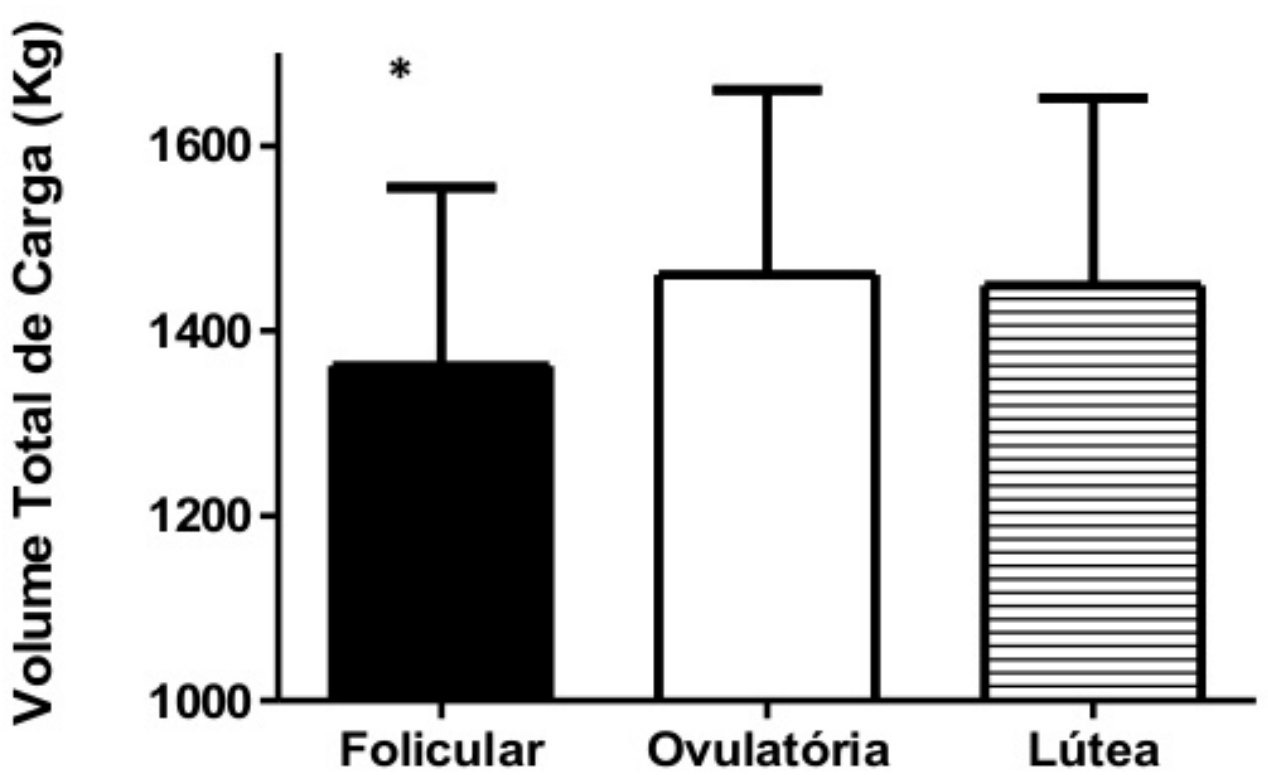

Figura 1: O volume total de carga levantada (Séries x Repetições x Carga $[\mathrm{kg}]$ ) nas fases folicular, ovulatória e lútea. * significativamente menor $(\mathrm{p}<0,05)$ em relação à fase folicular e lútea $(\mathrm{n}=20)$. Dados são média \pm desvio padrão.

\section{Discussão}

O objetivo deste estudo foi avaliar a influência das três fases do ciclo menstrual na performance de força muscular dos membros superiores durante treinamento de força em mulheres já treinadas e usuárias de anticoncepcionais. Nosso principal achado foi que ao longo do período observado ocorreu queda na performance muscular apenas no período folicular.

Durante o ciclo menstrual observa-se variações nas concentrações dos hormônios esteróides femininos (LEBRUN, 1993). No início da fase folicular os níveis de estrógeno e progesterona estão baixos, culminado com o início da menstruação, posteriormente à concentração de estrógeno aumenta, atingindo seu pico pouco antes da fase ovulatória (JANSE DE JONGE, 2003). Sintomas menstruais como dismenorréia, cansaço, retenção de liquido e ganho de peso são postulados por terem efeitos adversos sobre a performance de atletas e pessoas ativas (LEBRUN, 1993). O treinamento de força é empregado com o objetivo de melhorar a capacidade de força e potência muscular por meio de fatores neurais e musculares (KRAEMER; RATAMESS, 2004). Assim, a performance neuromuscular pode ser influenciada por fatores psicológicos como motivação e disposição para a realização do 
esforço máximo ou extenuante, fator que pode ter contribuído com a variação da força muscular encontrada no presente estudo.

Estudos realizados por Dias e Simão (2005) e Loureiro et al. (2011), indicam que não existe perda significativa da força muscular nas diferentes fases do ciclo menstrual, quando avaliadas pelo o teste de 10RM em membros inferiores e superiores. Por outro lado, resultados obtidos por Simão et al. (2007) sugerem uma redução da capacidade de produção de força muscular para membros inferiores avaliadas pelo teste de $8 \mathrm{RM}$, quando realizados no primeiro dia da fase menstrual.

Normalmente tais estudos (DIAS; SIMÃO, 2005; SIMÃO et al., 2007; LOUREIRO et al., 2011), buscam avaliar a produção de força muscular nas diferentes fases do ciclo menstrual em dias pontuais, não controlando a rotina de treinamentos das voluntárias. Metodologicamente o que difere de nosso estudo, que avaliou a performance de força muscular em diferentes fases, no entanto, durante o processo de treinamento controlado.

De acordo com os resultados do presente estudo, as variações hormonais dentro do ciclo menstrual, em especial no período folicular, promoveu queda significativa na capacidade de força muscular em membros superiores, culminando na diminuição do volume total de carga levantada. Desta forma, as baixas taxas hormonais presentes no início do período folicular, podem influenciar a progressão de cargas durante o processo de treinamento.

Entre as limitações encontradas no presente estudo, o não controle das fases do ciclo menstrual, por meio de análises das concentrações séricas de estrógeno e progesterona, não nos permite aprofundar a discussão em termos da influência da razão dos hormônios estrógeno e progesterona sobre a performance muscular. Contudo, o fato das voluntárias serem usuárias de anticoncepcionais, promove ciclos regulares de 28 dias.

Apesar das limitações metodológicas, o presente estudo revela dados importantes que devem ser levados em consideração durante a elaboração de programas de treinamento em mulheres usuárias de medicamentos anticoncepcionais, para que se torne mais eficiente o controle das cargas e prescrição de sessões de treinamento. 


\title{
Conclusões
}

Os resultados do presente estudo sugerem que as diferentes fases do ciclo menstrual de mulheres usuárias de anticoncepcionais pode influenciar o desempenho de força muscular em membros superiores, com prejuízo da mesma durante a fase folicular. Tais alterações provavelmente decorrem de variações hormonais e deveriam ser consideradas no planejamento do processo de treinamento de mulheres atletas e não atletas.

\section{THE FOLLICULAR PHASE INFLUENCE THE MUSCULAR PERFOR- MANCE DURING THE STRENGTH TRAINING}

\begin{abstract}
The aim of this study was to evaluate the influence of the menstrual cycle on the performance of muscle strength. Twenty trained women and contraceptive users participated as volunteers. The training lasted 26 days (12 sessions - Monday, Wednesday and Friday) and involved only the exercise bench press (6 sets of 10 repetitions at $80 \% 1 \mathrm{RM}$ and pauses of 60 seconds between sets). Tor compare muscle performance, was considered the total volume of load lifted (sets x repetitions $x$ load) concerning the sessions: 1th and 3th day (folicular phase), 8th and 12th day (ovulatory phase), 15th and 26th day (luteal phase). Our results suggest a significant reduction in the total volume of load only in the follicular phase. In conclusion, follicular phase influenced the performance of muscle strength.

Keywords: Menstrual Cycle. Muscle Strength. Resistance Training.

\section{LA FASE FOLICULAR INFLUIR EN EL RENDIMIENTO MUSCULAR EL ENTRENAMIENTO DE FUERZA}

\section{Resumen}

El objetivo de este estudio fue evaluar la influencia del ciclo menstrual en el rendimiento de la fuerza muscular. Veinte mujeres capacitadas y los usuarios de anticonceptivos participaron como voluntarios. El entrenamiento duró 26 días (12 sesiones - Lunes, miércoles y viernes) que limita el ejercicio de press de banca (6 series de 10 repeticiones al $80 \%$ de 1 RM y 60 segundos pausas entre series). Para comparar el rendimiento muscular, se consideró el volumen total de carga elevada (series $\mathrm{x}$ repeticiones x carga) sesiones en relación con: $1^{\circ}$ y $3^{\circ}$ día (fase folicular), $8^{\mathrm{a}}$ y 12 a (fase ovulatoria) y $15^{\circ} 26^{\circ}$ día (fase lútea). Nuestros resultados mostraron una reducción significativa en el volumen total de carga sólo en la fase folicular. En conclusión, la fase folicular influenciado el rendimiento de la fuerza muscular.

Palabras clave: Ciclo Menstrual. Fuerza Muscular. Entrenamiento de Resistencia. 


\section{Referências}

BENNELL, K. S.; WHITE, S.; CROSSLEY, K. The oral contraceptive pill: a revolution for sportswomen? British Journal Sports Medicine, Loughborough, v. 33, n. 4, p. 231-8, 1999.

BROWN, L. E.; WEIR, J. P. ASEP Procedures recommendation I: accurate assessment of muscular strength and power. Journal of Exercise Physiology online, Diluth, v. 4, n. 1, p. 1-24, 2001.

BURROWS, M.; PETERS, C. E. The influence of oral contraceptives on athletic performance in female athletes. Sports Medicine, Auckland, v. 37, n. 7, p. 557-74, 2007.

CONSTANTINI, N. W;. DUBNOV, G.; LEBRUN, C. M. The menstrual cycle and sport performance. Clinics in Sports Medicine, Philadelphia, v. 24, n. 2, p. e51-82, 2005.

DESCHENES, M. R.; KRAEMER, W. K. Performance and physiologic adaptations to resistance training. American Journal of Physical Medicine \& Rehabilitation, Philadelphia, v. 81, n. 11, p. S3-16, 2002.

DIAS, I.; SIMÃO, R. Efeitos das diferentes fases do ciclo menstrual em um teste de 10RM. Fitness \& Performance Journal, Rio de Janeiro, v. 4, n. 5, p. 288-92, 2005.

JANSE DE JONGE, X. A. Effects of the menstrual cycle on exercise performance. Sports Medicine, Auckland, v. 33, n. 4, p. 833-51, 2003.

KRAEMER, W. J.; RATAMESS, N. A. Fundamentals of resistance training: progression and exercise prescription. Medicine and Science in Sports and Exercise, Madison, v. 36, n. 4, p. 647-88, 2004.

LEBRUN, C. M. Effect of the different phases of the menstrual cycle and oral contraceptives on athletic performance. Sports Medicine, Auckland, v. 16, n. 6, p. 400-30, 1993.

LOUREIRO, S. et al. Efeitos das diferentes fases do ciclo menstrual no desempenho da força muscular em 10RM. Revista Brasileira de Medicina do Esporte, São Paulo, v. 17, n. 1, p. 22-25, 2011. 
OOSTHUYSE, T.; BOSCH, A. N. The effect of the menstrual cycle on exercise metabolism: implications for exercise performance in eumenorrhoeic women. Sports Medicine, Auckland, v. 40, n. 3, p. 20727, 2010.

SIMÃO, R. et al. Variações na força muscular de membros superior e inferior nas diferentes fases do ciclo menstrual. Revista Brasileira de Ciência e Movimento, Brasília v. 15, n. 3, p. 47-52, 2007.

Recebido em: $14 / 08 / 2012$

Revisado em: 17/02/2013

Aprovado em: 29/06/2013

Endereço para correspondência

alexhcrisp@gmail.com

Alex Harley Crisp

Universidade Metodotista de Piracicaba

Laboratório Peformance Humana.

Rodovia do Açúcar Km 156

Taquaral

13400911 - Piracicaba, SP - Brasil 